\title{
Early Onset Post-Radiation Cutaneous Angiosarcoma of the Breast with Features of Atypical-Vascular Lesion: A Diagnostic Pitfall and Its Biologic Significance
}

\author{
John B. Wojcik', Brian J. Czerniecki'2,3, Paul J. Zhang1* \\ ${ }^{1}$ Department of Pathology and Laboratory Medicine, University of Pennsylvania Perelman School of Medicine, \\ Hospital of the University of Pennsylvania, Philadelphia, USA \\ ${ }^{2}$ Department of Surgery, University of Pennsylvania Perelman School of Medicine, Philadelphia, USA \\ ${ }^{3}$ Rena Rowen Breast Center, Hospital of the University of Pennsylvania, Philadelphia, USA \\ Email: ${ }^{*}$ jz@@mail.med.upenn.edu
}

Received 21 April 2015; accepted 1 June 2015; published 4 June 2015

Copyright $@ 2015$ by authors and Scientific Research Publishing Inc.

This work is licensed under the Creative Commons Attribution International License (CC BY). http://creativecommons.org/licenses/by/4.0/

(c) () Open Access

\begin{abstract}
Vascular proliferations may arise in the breast following radiation treatment for a primary breast adenocarcinoma. A post-radiation vascular proliferation can usually be classified as angiosarcoma or as an atypical vascular lesion (AVL). Angiosarcomas with a "low-grade" morphology, behave aggressively but exhibit substantial histomorphologic overlap with AVLs, which have a generally benign clinical course. We present a case of a post-radiation angiosarcoma of the breast with histologic features that mimic an atypical vascular lesion and discuss this challenging differential diagnosis. In addition to histologic mimicry, the lesion exhibited only patchy amplification of the avian myelocytomatosis viral oncogene homolog (MYC) gene by present fluorescence in-situ hybridization (FISH), and patchy MYC overexpression by immunohistochemistry. These features further complicate the distinction between AVL and angiosarcoma, and would be particularly problematic on a small biopsy. We believe that the morphologic and immunohistochemical overlap between these entities is suggestive of a biologic spectrum, and thus that, at least in some instances, angiosarcoma may arise from a pre-existing AVL or AVL-like lesion.
\end{abstract}

\section{Keywords}

Angiosarcoma, Atypical Vascular Lesion, MYC Amplification, Radiation Associated Sarcoma

\footnotetext{
${ }^{*}$ Corresponding author.

How to cite this paper: Wojcik, J.B., Czerniecki, B.J. and Zhang, P.J. (2015) Early Onset Post-Radiation Cutaneous Angiosarcoma of the Breast with Features of Atypical-Vascular Lesion: A Diagnostic Pitfall and Its Biologic Significance. Case Reports in Clinical Medicine, 4, 203-208. http://dx.doi.org/10.4236/crcm.2015.46041
} 


\section{Introduction}

There have long been reports of vascular neoplasia arising in the female breast following therapy for a primary breast carcinoma. The earliest of these reports focused on chronic lymphedema-associated angiosarcoma-the so-called Stewart-Treves syndrome [1]. More recently, there have been numerous cases and series illustrating the clinicopathologic features of cutaneous angiosarcoma arising following breast-conserving surgery with adjuvant radiation therapy [2] [3]. Post-radiation vascular proliferations, however, are not limited to angiosarcoma. Beginning with the earliest description roughly 20 years ago, it was noted that a subset of post-radiation vascular proliferations had clinicopathologic features that were distinct from those of the typically aggressive angiosarcomas [4]. These were termed atypical vascular lesions (AVL). Since the initial reports, other case studies have borne out this observation, though the distinction between AVL and angiosarcoma remains a challenge due to significant overlap between the entities [5]-[7]. We present a case study that highlights the challenge of distinguishing between these entities on a morphologic basis. We believe that it also provides evidence for a biologic relationship between the two entities, and thus that, at least in some instances, angiosarcoma may arise from a pre-existing AVL or AVL-like lesion.

\section{Case Presentation}

A 73-year-old female presented with a new violaceous macular "rash" in the field of radiation treatment for a prior invasive ductal carcinoma of the left breast two years ago. The invasive ductal carcinoma was treated with breast conservative excision, sentinel lymph node biopsy, adjuvant chemotherapy and whole breast radiation. As a consequence of therapy she experienced mild lymphedema of the breast beginning shortly after radiation therapy, but was otherwise well. A punch biopsy of the lesion was obtained, and the initial diagnosis rendered at an outside institution was reported to be atypical vascular lesion. Subsequent MRI studies revealed skin thickening and enhancement adjacent to the location of the biopsy. She underwent an excision of the area surrounding the biopsy, which included both the visible rash and the area with abnormal MRI findings. On excision, the specimen was found to contain an atypical vascular proliferation involving the superficial and deep dermis with the same features as the biopsy, but also areas of multi-layered endothelium, mild to moderate nuclear atypia and increased mitotic activity. Due to the presence of these features, the tumor was diagnosed as angiosarcoma at the outside institution. Because of the incomplete excision, the patient was referred to our institution for a re-excision, and elected to undergo bilateral mastectomy with wound coverage using autologous free flap.

The excised specimen contained a healing scar and mild erythema, but lacked clearly identifiable cutaneous lesions suggestive of residual angiosarcoma (Figure 1). The soft tissues deep to areas of scar exhibited fat necrosis and hemorrhage representing the prior excision site. The skin was sampled extensively, and similar vascular findings were identified diffusely throughout the breast skin, along an area measuring more than $10 \mathrm{~cm}$ in greatest dimension. The residual tumor was only appreciable under microscopic examination and consisted of

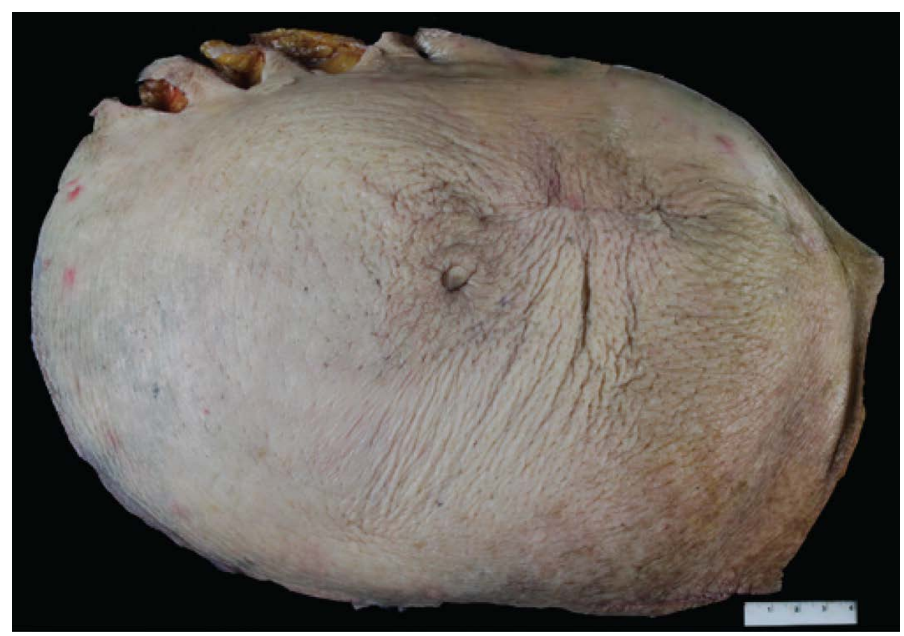

Figure 1. Mastectomy specimen. The scar from the prior excision is present, but no residual cutaneous lesion is grossly identifiable. 
scattered poorly defined areas of thin walled vessels, some angulated and some anastomosing, both within the superficial and deep dermis. The vessels in the superficial dermis were ectatic, and lined by a single layer of minimally atypical endothelial cells with condensed chromatin and no evident nucleoli (Figure 2(A) and Figure (B)). They focally formed complex, anastomosing channels that surrounded adnexal structures and pilar muscles in a sinusoidal pattern, and formed small papillae lined by a single layer of endothelium (Figure 2(C)). Deeper aspects of the lesion contained compressed, irregular slit-like vascular spaces, again lacking atypical cytologic features. In both the superficial and deep aspects, the vessels were accompanied by a lymphocyte-predominant mild inflammatory infiltrate. Extension into the subcutis was not seen. Nowhere in the extensively sampled excision were there overt features of malignancy in the form of marked atypia, multi-layering or solid growth or

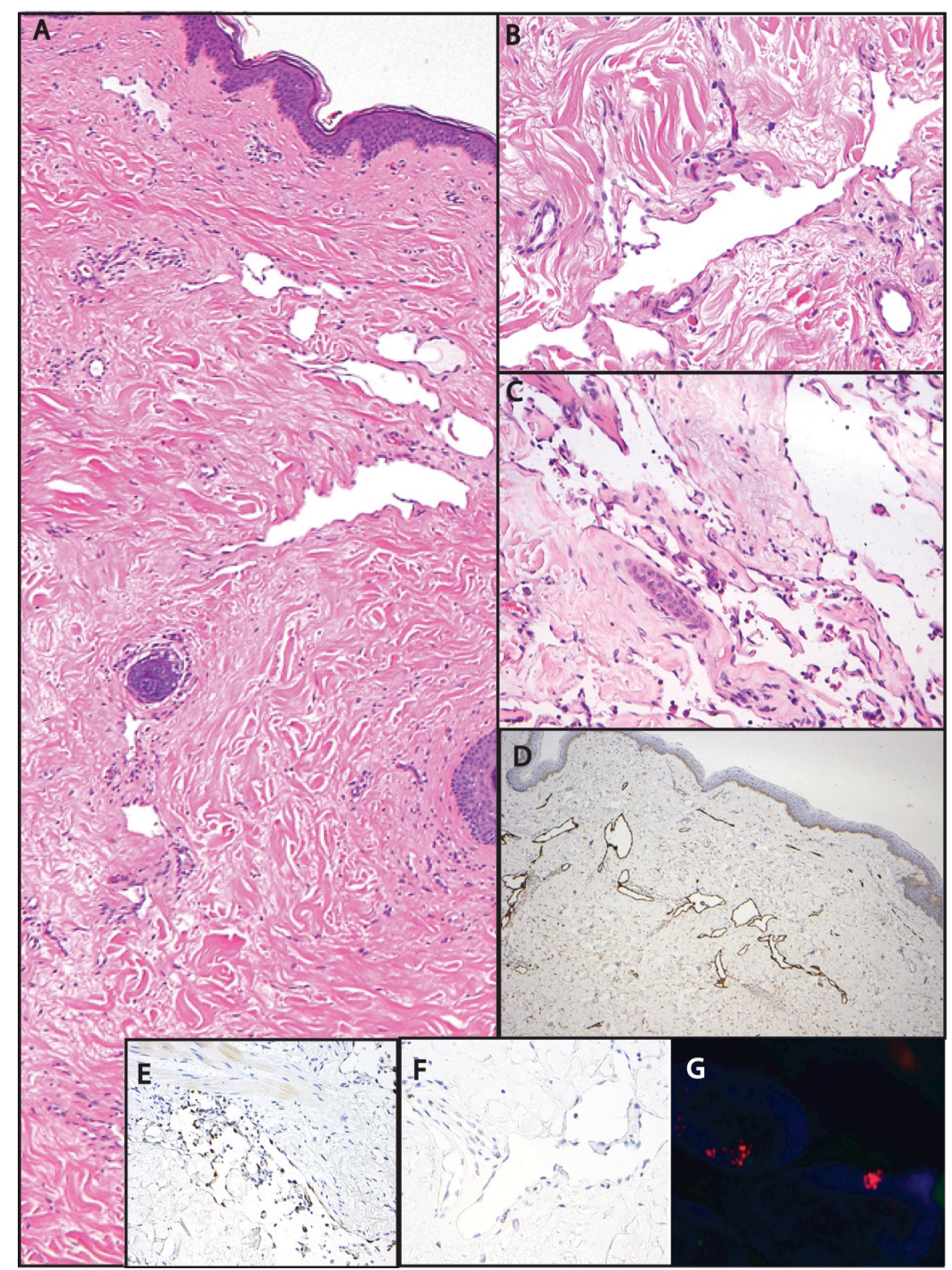

Figure 2. Histology of post-radiation angiosarcoma in a background of diffuse AVL-like changes. (A)-(C) Low-power view (A) highlights a dermal vascular proliferation of irregular, anastomosing vessels. On higher power (B)-(C), the lesion contains a single layer of minimally atypical endothelium, lacking nucleoli, multi-layering or mitotic activity, though focal stromal papillae, lined by a single-layer of endothelial cells, project into the abnormal vessels. Immunohistochemistry for D240 (D) shows a lymphatic phenotype, and highlights the extent of the vascular proliferation. Immunohistochemical staining for MYC (E)-(F) shows only focal robust expression, consistent with the focal amplification of the MYC locus demonstrated on fluorescence in situ hybridization ((G), red probe). 
mitotic activity. An immunohistochemical stain for D240 was diffusely positive in the atypical vascular proliferation, and helped identify the extent of the dermal involvement (Figure 2(D)). cMYC immunohistochemical stain showed focal strong positivity in the vessels directly adjacent to the prior excision (Figure 2(E) and Figure 2(F)). In this location, MYC amplification was confirmed by fluorescence in situ hybridization in some of the cells with MYC nuclear reactivity but not all the lesional endothelial cells. The specimen margins were uninvolved by the atypical vascular proliferation. Except for the diffuse involvement of the skin and focal MYC amplification/expression, the morphologic features of the lesion in the excision were entirely within the histologic spectrum of AVL. After six months of follow-up, there is no evidence of recurrent or metastatic disease.

\section{Discussion}

There are a number of interesting and illustrative features of this case that warrant particular attention, and highlight the challenges posed in the identification and classification of low-grade vascular lesions, particularly in the post-radiation setting. The most critical distinction in this context is between a post-radiation atypical vascular lesion (AVL) and post-radiation angiosarcoma. Radiation-associated angiosarcomas occur with a median latency period of 5 - 6 years after breast radiation, and have a poor prognosis, with aggressive local behavior and substantial metastatic potential [2] [8]. AVLs have a similar or slightly shorter latency period, varying from 3.5 5.5 years, depending on the series, but with a greater fraction of cases presenting within 1 - 2 years of therapy [4] [7]-[10]. Because of its generally benign clinical behavior, AVL may be excised more conservatively, whereas radiation-associated angiosarcoma may both recur locally and metastasize even following wide excision. Thus, distinguishing between these entities has important therapeutic implications. This challenge is compounded by the suggestion that, while most AVLs have a benign clinical course, some may undergo transformation into angiosarcoma [5] [7]. Currently, there is a lack of morphologic criteria to reliably predict which AVL may progress. Indeed, it is possible that they are not entirely discrete entities, but rather exist on a spectrum.

In the current case, the short interval between the original breast excision and the development of the lesion ( 23 months) might lead one to favor AVL, which has a shorter median latency period. Nonetheless, there is wide variation and substantial overlap in the latency period between radiation and the development of either an AVL or an angiosarcoma. Thus, while AVL have a shorter median latency period, this is not a reliable discriminator between AVL and angiosarcoma.

The histologic findings of the current case warrant particular attention. These features highlight the overlap between AVL and angiosarcoma, and are suggestive of a biologic relationship between these entities. Both the original and the subsequent descriptions of AVL noted a number of features useful in the distinction between AVL and angiosarcoma [4] [8]. AVLs were circumscribed lesions and typically lacked involvement of the subcutis, intraluminal red blood cells, extravasated blood, significant atypia, multi-layering, prominent nucleoli and mitotic activity. Relative to angiosarcomas, AVLs were more likely to exhibit areas of chronic inflammation. In this case, in both the biopsy and final excision, the histologic findings were almost entirely in keeping with those typically associated with AVL. The lesion consisted of a haphazard arrangement of thin-walled vessels that lacked the typical features of angiosarcoma. The sole histologic parameter that favored a diagnosis of angiosarcoma was the diffuse, infiltrative nature of the lesion. It has long been recognized that there is substantial histologic overlap between AVL and angiosarcoma with low-grade histologic features. In this case, the distinction required a wide excision, in which the most significant parameter (infiltration) was apparent.

In addition to infiltration, there was other evidence for a diagnosis of malignancy in the form of immunohistochemical and in-situ hybridization showing MYC amplification. In the past several years, MYC amplification has received considerable attention as a diagnostic marker of post-irradiation angiosarcoma [11]. Newer data, however, suggests that MYC amplification can be seen in both post-radiation and de novo angiosarcoma [12] [13]. Conversely, MYC amplification is not an invariable feature of either primary or secondary angiosarcoma, so its absence does not exclude the diagnosis. Nonetheless, in reports to date, MYC amplification has consistently been associated with malignancy, and is reportedly a reliable discriminator between AVL and radiationassociated angiosarcoma [14]. The current case showed areas with MYC amplification, a feature helpful in arriving at the diagnosis of angiosarcoma. However, abnormal MYC amplification and over expression were only detected in small population of the lesional cells. This heterogeneity in MYC amplification could have significant implications for the biopsy diagnosis of AVL, suggesting that negative FISH or IHC detection might not completely exclude the diagnosis of angiosarcoma. 
Another immunohistochemical feature worth noting in the current case is the lymphatic immunophenotype of the atypical vascular proliferation. AVLs have been classified as lymphatic or vascular types principally on the basis of immunophenotype, with the lymphatic-type AVLs (ltAVL) representing the majority, and expressing D240 [9]. In a large series, those with a lymphatic phenotype behaved less aggressively than those with a vascular phenotype [7]. The lesion in this case showed a lymphatic phenotype and thus, on the initial biopsy, would have been classified as an ltAVL. On this basis, the lesion may have been predicted to behave less aggressively. However, while of potential use in the subclassification of AVLs, D240 is less useful in distinguishing AVL from angiosarcoma, since angiosarcomas may express D240 [15]. Thus, D240 staining does not seem to constitute strong evidence that the angiosarcoma arose from a ltAVL. Other features of the case, however, lead us to favor this type of progression. The histomorphology was indistinguishable from AVL throughout the entirety of the lesion. Moreover, MYC amplification, strong evidence in favor of malignancy, was only observed focally. All of this is suggestive of an angiosarcoma arising in an AVL, or AVL-like lesion. This would be consistent with other reports that cite angiosarcomas arising from an AVL [7].

\section{Conclusion}

We have presented a case of angiosarcoma that we feel, based on histomorphology and the focal nature of MYC amplification, is suggestive of anangiosarcoma arising from an atypical vascular lesion. It also serves as an important illustration of the substantial morphologic overlap of histologically low-grade vascular lesions. It is important to bear this in mind, especially in the context of a large lesion that is only sparsely sampled in a core biopsy. In a lesion that is clinically large, or in which the microscopic features (e.g. diffuse growth pattern) suggest a larger lesion than that seen clinically, one should be extremely cautious with a diagnosis of AVL, even in the context of negative MYC IHC or FISH studies. In the current case, evidence for malignancy was subtle and focal, and only evident upon wider excision. Care must be taken to avoid a misdiagnosis of angiosarcoma as AVL since this could have major therapeutic and prognostic significance.

\section{References}

[1] Stewart, F.W. and Treves, N. (1948) Lymphangiosarcoma in Postmastectomy Lymphedema; a Report of Six Cases in Elephantiasis Chirurgica. Cancer, 1, 64-81. http://dx.doi.org/10.1002/1097-0142(194805)1:1<64::AID-CNCR2820010105>3.0.CO;2-W

[2] Billings, S.D., McKenney, J.K., Folpe, A.L., Hardacre, M.C. and Weiss, S.W. (2004) Cutaneous Angiosarcoma Following Breast-Conserving Surgery and Radiation: An Analysis of 27 Cases. American Journal of Surgical Pathology, 28, 781-788. http://dx.doi.org/10.1097/01.pas.0000126055.33916.0b

[3] Mentzel, T., Schildhaus, H.U., Palmedo, G., Buttner, R. and Kutzner, H. (2012) Postradiation Cutaneous Angiosarcoma after Treatment of Breast Carcinoma Is Characterized by MYC Amplification in Contrast to Atypical Vascular Lesions after Radiotherapy and Control Cases: Clinicopathological, Immunohistochemical and Molecular Analysis of 66 Cases. Modern Pathology, 25, 75-85. http://dx.doi.org/10.1038/modpathol.2011.134

[4] Fineberg, S. and Rosen, P.P. (1994) Cutaneous Angiosarcoma and Atypical Vascular Lesions of the Skin and Breast after Radiation Therapy for Breast Carcinoma. American Journal of Clinical Pathology, 102, 757-763.

[5] Brenn, T. and Fletcher, C.D. (2006) Postradiation Vascular Proliferations: An Increasing Problem. Histopathology, 48, 106-114. http://dx.doi.org/10.1111/j.1365-2559.2005.02293.x

[6] Lucas, D.R. (2009) Angiosarcoma, Radiation-Associated Angiosarcoma, and Atypical Vascular Lesion. Archives of Pathology and Laboratory Medicine, 133, 1804-1809.

[7] Patton, K.T., Deyrup, A.T. and Weiss, S.W. (2008) Atypical Vascular Lesions after Surgery and Radiation of the Breast: A Clinicopathologic Study of 32 Cases Analyzing Histologic Heterogeneity and Association with Angiosarcoma. American Journal of Surgical Pathology, 32, 943-950. http://dx.doi.org/10.1097/PAS.0b013e31815bf8fe

[8] Brenn, T. and Fletcher, C.D. (2005) Radiation-Associated Cutaneous Atypical Vascular Lesions and Angiosarcoma: Clinicopathologic Analysis of 42 Cases. American Journal of Surgical Pathology, 29, 983-996.

[9] Fraga-Guedes, C., Gobbi, H., Mastropasqua, M.G., Rocha, R.M., Botteri, E., Toesca, A. and Viale, G. (2014) Clinicopathological and Immunohistochemical Study of 30 Cases of Post-Radiation Atypical Vascular Lesion of the Breast. Breast Cancer Research and Treatment, 146, 347-354. http://dx.doi.org/10.1007/s10549-014-3020-9

[10] Gengler, C., Coindre, J.M., Leroux, A., Trassard, M., Ranchere-Vince, D., Valo, I., Michels, J.J. and Guillou, L. (2007) Vascular Proliferations of the Skin after Radiation Therapy for Breast Cancer: Clinicopathologic Analysis of a Series in Favor of a Benign Process: A Study from the French Sarcoma Group. Cancer, 109, 1584-1598. 
http://dx.doi.org/10.1002/cncr.22586

[11] Manner, J., Radlwimmer, B., Hohenberger, P., Mossinger, K., Kuffer, S., Sauer, C., Belharazem, D., Zettl, A., Coindre, J.M., Hallermann, C., Hartmann, J.T., Katenkamp, D., Katenkamp, K., Schoffski, P., Sciot, R., Wozniak, A., Lichter, P., Marx, A. and Strobel, P. (2010) MYC High Level Gene Amplification Is a Distinctive Feature of Angiosarcomas after Irradiation or Chronic Lymphedema. American Journal of Pathology, 176, 34-39. http://dx.doi.org/10.2353/ajpath.2010.090637

[12] Shon, W., Sukov, W.R., Jenkins, S.M. and Folpe, A.L. (2014) MYC Amplification and Overexpression in Primary Cutaneous Angiosarcoma: A Fluorescence In-Situ Hybridization and Immunohistochemical Study. Modern Pathology, 27, 509-515. http://dx.doi.org/10.1038/modpathol.2013.163

[13] Ginter, P.S., Mosquera, J.M., MacDonald, T.Y., D’Alfonso, T.M., Rubin, M.A. and Shin, S.J. (2014) Diagnostic Utility of MYC Amplification and Anti-MYC Immunohistochemistry in Atypical Vascular Lesions, Primary or RadiationInduced Mammary Angiosarcomas, and Primary Angiosarcomas of Other Sites. Human Pathology, 45, 709-716. http://dx.doi.org/10.1016/j.humpath.2013.11.002

[14] Hafner, C. (2013) MYC Amplification Distinguishes between Atypical Vascular Lesion and Radiation-Induced Angiosarcoma of the Breast. Der Hautarzt, 64, 533-534. http://dx.doi.org/10.1007/s00105-013-2602-5

[15] Kahn, H.J., Bailey, D. and Marks, A. (2002) Monoclonal Antibody d2-40, a New Marker of Lymphatic Endothelium, Reacts with Kaposi's Sarcoma and a Subset of Angiosarcomas. Modern Pathology, 15, 434-440. http://dx.doi.org/10.1038/modpathol.3880543 